\title{
Cenno di alenni studi sul latte
}

\author{
fatti da Fr. SelmI.
}

In questo scritto mi terrò stretto ad una rapida esposizione, chiara per quanto da me si potrà rendere, degli esperimenti che instituii nell' inverno trascorso sul latte, affine di conoscere la vera causa onde si rappiglia per l' influenza del presame. L' opinione che attualmente vige presso i chimici, dopo le cose pubblicate dallo Scherer, dal Rochleder e da altri, adottata e promulgata eziandio dal celebre Liebig è la seguente: che il presame coauguli il latte, perchè agendo sulla lattina la trasforma in acido lattico, il quale impadronendosi dell' alcali libero che nel latte mantiene sciolta la caseina, guasta cosi il caseato alcalino solubile, e costringe la sostanza proteifera a precipitare nello stato $d^{\prime}$ insolubilità .

1.0 Tale opinione non è stata riconfermata dalle mie esperienze; poichè sono giunto a coagulare il latte munto di fresco e decisamente alcalino con buona infusione neutra di presame, senza che il siero ed il coagulo avessero perduta la reazione alcalina; ottenendo il rapprendimento in pari modo anche col latte reso alcalino a bella posta per mezzo del carbonato di soda, spingendo l'alcalinamento fino al punto da raccogliere un coagulo caseico ridissolubile nell' acqua pura per l'effetto della soda rimastagli aderente. Ma a meglio convincere che la coagulazione non proviene, nè dalla formazione dell' acido lattico, nè dall' inacidimento del latte, precipitai porzioni diverse di questo liquido, cogli acidi tartarico, acetico, ossalico, cloridrico allungati; ridisciolsi $i$ singoli precipitati mediante un esuberanza dell' acido adoperato, e poscia, tentai la loro coagulazione, che ottenni, servendomi tanto dell' infusione di presame, quanto di pezzetti della membrana dello slomaco di vitello. Dubitando che la presenza dei sali calcari esistenti nel presame quantunque disciolti Cim. An. IV. 


\section{8}

dagli acidi liberi, potessero indurre in errore col produrre un falso coagulo, istituii indagini comparative, dalle quali fu dileguato qualsivoglia dubbio.

La caseína sciolta sola negli acidi sunnominati resiste di più al potere coagulatore del presame che non faccia il latte ridisciolto dagli acidi stessi, onde si vede che le materie commiste seco lei nel latte, coadiuvano per la presenza loro l' azione coagulatrice, ovvero che sciolta separatamente assume attitudini alquanto diverse da quelle che possiede quando trovasi nel latte.

2. Ho riconosciuto che il presame eccita ben presto un moto di fermentazione nella caseina. Unito al siero di latte, vi produce un sollecito inacidimento che rapido fino ad un dato puuto cresce, e poscia diminuisce nel suo generarsi, sebbene una porzione solo della lattina sia trasformata; il siero solo posto in eguali circostanze s' acidifica a gradi crescendo meno rapidamente, ma sempre aumentando fino al punto che raggiunga la forza acida del siero misto a presame. Se si pone a confronto dei due vasi contenenti $i$ due sieri, un terzo vaso con soluzione acquosa di lattina associata ad energica infusione di presame, si nota che mentre $i$ primi si caricano in copia dell' acido lattico, il terzo liquido s' inacidisce appena, sebbene si adoperi presame molto energico. Da ció e da altri fatti fui guidato a concludere: che l'azione, del presame si esercita sulle materie solfo-azotate, pel cui intermedio si metamorfosa la lattina, e che il presame puro, se trasforma alcun poco la lattina unitagli, ciò forse effettua per la presenza delle sostanze estrance azotate alle quali va naturalmente mescolato. Non si creda poi, che il rallentarsi l' inacidimento del primo siero misto a presame derivi da mancanza di lattina già metamorfosata, poichè il risultamento riesce identico quando si pone in opera siero a cui artificialmente sia stato associato certa dose di lattina.

$3 .^{0}$ Il presame fa conoscere d' essere atto a riprodursi 
col mezzo delle sostanze azotate, quando sia seco loro unito con un principio che mantenga la fermentazione lontana dell' infracidimento. Di fatto mescolando infusione di presame, e siero di latte, infusione di presame e soluzione di caseina o d'albumina con zucchero di latte, infusione di presame e soluzione dello zucchero di latte solo, si hanno liquidi coagulatori, dopo alcuni giorni, nei quali la virtù rappigliatrice appare superiore nel primo, e nel secondo assai più che nel terzo. Se manca la lattina e si abbia il presame colla caseina sciolta o l'albumina, queste rimangono ben presto tradotte a putrefazione, e l'efficacia del presame diminuisce di pari passo con celerità.

4.0 Ma il latte puro, scevro da presame può tramutarsi in presame se venga collocato in istufa o previamente sia stato salato; il medesimo latte non salato non da luogo a produzione del principio rappigliatore. Le soluzioni di caseina e d'albumina, pure, collo scomporsi o guastarsi divengono atte a coagulare il latte come si fossero presame, abbenchè agiscano in ciò debolmente.

$5 .^{\circ}$ La virtù coagulante del presame opera a freddo sul lalte come fa a caldo, tranne della differenza che esige parecchi giorni di tempo per dare compimanto al fenomeno. In capo di tre giorni in cui la temperie non sorpassò $i+3^{0} \mathrm{~K}$, coagulai del latte, con formazione di grumi caseici e di siero limpido in quella guisa che si hanno lavorando a caldo. La coagulazione a freddo si ottiene col latte alcalinulato, senza che questo perda la reazione tramutatrice sulla carta rossa del tornasole, fatto che riconferma quanto fu raccontato in sul principio.

$6 .^{\circ}$ I sali a base alcalina, ossia il nitro, il solfato potassico, il solfato sodico, gli acetati delle stesse basi, il tartarato potassico neutro, il solfato bibasico di soda ritardano notabilmente, qual più qual meno, la coagulazione del latte operata col mezzo del presame. Il nitro ed i due solfati alcalini, se in dose discreta, impediscono to- 


\section{0}

talmente la coagnlazione, non senza qualche differenza dí azione poichè il solfato sodico altera le qualifà della caseina e la rende non più precipitabile coll'acido acetico, mentre gli altri due sali non ingenerano tale alterazione. Il tartrato potassico in leggier dose lascia produrre un debol coagulo; a proporzione maggiore impedisce il rappigliamento ordinario, mentre il liquido acquista vischiosità.

7. ${ }^{0}$ Egli è quindi a notarsi che il fosfato bibasico di soda, abbenchè adoprato in bư ona quantità non s' oppone totalmente alla coagulazione del latte col presame, mentre in tenuissima porzione impedisce alla caseina sola, seco lui disciolta, di costiparsi sotto l' influenza del principio rapprenditore. Ciò coincide col fatto conosciuto che la caseina sola col carbonato sodico riesce non rappigliabile.

8. ${ }^{\circ}$ Gli acidi minerali ed organici aggiunti al latte in dose tale che questo non si rappigli dai medesimi soltanto a $+50^{\circ}$ R., sollecitano la coagulazione col presame; l' attività col coagularsi è tale che il fenomeno può effettuarsi a freddo in una a due ore, ed a caldo in brevi minuti. Non tutti gli acidi adoprati in dosi equivalenti hanno eguale potere eccitatore del rappigliamento; l'acido lattico riesce il più energico di tutti, poscia seguono per ordine l'acetico, il nitrico, il cloridrico, il solforico, il citrico ec. Anche questo fatto dimostra che la coagulazione è indipendente dalla saturazione dell' alcali libero proprio al latte, giacchè la forza promovitrice della coagulazione, insita negli acidi, seconderebbe la loro affinità per le basi, e primo serebbe l' acido solforico, poi il cloridrico ec., ed ultimi l' acidi lattico ed acetico.

Allorquando a due eguali proporzioni dello stesso latte si sopraversa in una tanto d'acido che basti a trasfonderle la reazione acidula decisa, nell' altra il doppio di quantità, aggiungendo ad ambedue il presame, si nota che la coagulazione a caldo, si compie in ambedue senza grande diversità di tempo ed alle volte nel medesimo punto: 
ciò dimostra che gli acidi non coadiuvano il rappigliamento in modo proporzionale alle loro quantità.

9. Ma le osservazioni più curiose furono quelle che diressi ad investigare l'azione degli acidi da soli nel provocare a freddo il congrumarsi della caseina nel latte.

Io prego chi leggerà questo mio scritto a concedermi speciale attenzione per il presente paragrafo.

Volli conoscere in qual maniera gli acidi si comportino colla caseina nel rappigliarla da soli a freddo, e perciò preparai soluzioni dilute dei medesimi, composte in guisa che ad egual volume, ciascuna soluzione, contenesse un equivalente dell' acido. Cimentando poscia cogli acidi suddetti il latte trovai che il cloridrico ed il nitrico agirono con parità di forze ; l'acido citrico s'allontanò alquanto; l'acetico mostrò una meravigliosa diversità, poichè fù necessario adoprarne tre volte tanto degli altri acidi, per ottenere l' effetto desiderato. Ciò accadde col latte recente.

Col latte maturo le differenze cominciarono a diminuire notabilmente, di guisa che bastavano 2 equivalenti ed anche 1 1/2 d' acido acetico a produrre la congrumazione; col latte maturo assai, sparve ogni diversità e l' acido acetico spiegò tanta virtù coagulatrice quanta ne possederano gli altri acidi. Così era a cercare per qual ragione avvenissero tali fenomeni, perchè la maturità del kitte eccitasse le deboli forze dell' acido acetico al punto da renderlo eguale agli acidi $i$ più energici. Dopo lunghe e pazienti indagini falte sul latte perfettamente neutro, sul latte inacidito artificialmente, sul latte misto a presame disciolto, e poscia cimentato coll' acido acetico, ebbi ad accorgermi e ad assicurarmi, che gli acidi in genere posseggono due diversi periodi d'azione sulla caseina del latte; nel primo dei quali sembra, sono per dire, che la predispongano a raggrumarsi, mentre nel secondo, predispostala, ne effettuano la coagulazione. Nel primo periodo, che io chiano preparatorio, operano con forze dissimili, nel secondo, che io nomino completorio, agiscono 


\section{2}

con eguale energia. Difatto se aggiungasi tanto d'acido solforico, lattico e cloridrico a latte recente od anche mezzo maturo, per ridurlo ai termini del periodo preparatorio, vedesi l' acido acetico compiere il secondo periodo con quella forza onde si comporterebbe uno dei tre acidi sovrindicati. Ora si può spiegare chiaramente il perchè nel latte maturo l'acido acetico si mostri vigoroso: il latte nel mutarsi si carica di acido lattico e a tal grado che il periodo preparatorio rimane compiuto, laonde quando si assaggia coll' acido acetico, questi agisce con energia a somiglianza di qualsivoglia altro acido.

L'infusione di presame, può sottentrare agli acidi nel compiere l' uno o l'altro dei due periodi, nel che adopra con forze sempre uniformi. Perciò si hanno risultamenti diversi con quantità eguali di latte, d' acido, ed infusione di presame, a seconda che si aggiugne al latle l'acido od il presame in precedenza : acidificando prima il latte non basta più quella dose di presame a compiere la coagulazione che valse nel caso in cui si aggiungesse primo il presame sul latte, ed in appresso l' acido. La ragione è manifesta, giacchè l' acido posto in antecedenza soddisfacendo al periodo preparatorio svolge un potere minore di quello che manifesti, allorquando stillato in seguito del presame, trova il detto periodo già terminato, e non gli resta che a compiere il completorio.

$10 .^{\circ}$ I sali a base alcalina, alla temperatura in cui il latte bolle, lo coagulano, ma l'azione loro torna più vigorosa del doppio nel caso in cui gli si mescolino a freddo, avanti lo scaldamento, di quello che unendoli nell'atto in cui comincia a bollire. Lo zucchero invece opera in modo precisamente inverso dei sali a base alcalina.

11. Durante il rappigliamento del latte non si ha nè sviluppo di calorico, nè variazione nel volume della massa.

12. II latte intorpidisce singolarmente l'azione degli acidi e degli alcali fra loro, o sulle carte colorate. La curcuma non comincia a far palase gli alcali nel latte se 
non quando questi vi si trovino in copia; la carta rossa di toraasole corrisponde assai più squisitamente.

$13 .^{\circ}$ La caseina si discioglie nei sali a base alcalina, con facilità, dalle quali soluzioni riesce sempre separabile col mezzo dell' acido acetico. I liquidi salini molto concentrati ne sciolgono meno dei medesimi liquidi allungati, di guisa che con certi sali ho coagulata a freddo la caseina sciolta in un liquido salino diluto, con aggiungervi semplicemente o nuova dose di sale, ovvero un volume di soluzione concentratissima del sale medesimo. Scaldando tali soluzioni caseiche, la coagulazione comincia a $+30^{\circ}$ R., ed alle volte ad uu grado anche inferiore, e si compie $a+60^{\circ}$. Lasciando il liquido salino unito alla sostanza coagulata questa a poco a poco vi si discioglie.

14.0 Il latte abbandonato in vasi metallici intacca $i$ metalli, e ne scioglie parecchi in abbondanza. Intacca pure il solfo e provoca un notabile svolgimeuto d' idrogeno solforato, fenomeno che ho osservato ripetersi in tutte le sostanze organiche azotate, miste al solfo, quando cominciano ad alterarsi. Le sostanze organiche non azotate sono inette a promuovere la formazione dell' idrogeno solforato col solfo.

Mi riserbo a trattare più diffusamente intorno a tutte le cose riferite nel lavoro che sto per pubblicare sul latte, ove mi farò a discutere eziandio la parte teorica ed a dedurne quelle applicazioni pratiche al caseifico che paionmi derivare dalle osservazioni da me istituite.

\section{Sopra una Impressione manifestatasi gulla tavoletta di un quadro}

\section{Lettera al Prof. Lugi Pachotri.}

\section{Prof. Carissimo}

Dopo gli esperimenti e le ricerche che insieme e col Sig. Luigi Ridolfi facemamo intorno ai fenomeni cosi defli pel Moser, io non avrei creduto, adesso che son già scorsi 